\title{
Caracterización de genotipos de papa de Europa y Latinoamérica por resistencia a tizón y propiedades culinarias
}

\author{
J. Gabriel $^{1}$, F. Forqueda ${ }^{1}$, G. Plata ${ }^{1}$, E. Fernández-Northcote ${ }^{2}$
}

\begin{abstract}
RESUMEN
El objetivo de la presente investigación fue caracterizar 34 clones y/o variedades de papa de origen europeo y latinoamericano por su resistencia a tizón (Phytophthora infestans) y sus propiedades culinarias bajo condiciones de campo y laboratorio en tres campañas agrícolas. Los genotipos India (testigo), Chota Ñawi, Cordillera, Robusta, Jaspe y Yema de huevo (phu), mostraron altos niveles de resistencia en campo con baja Area Bajo la Curva de P. infestans (ABCPPI = 203-541). Los genotipos 387660-10, Torridón, SH-83-92-488, 90-145-6, 94-70-2502, 89T-117-10, Pampeana, DB-226(70) y Pollerita-V mostraron buenos niveles de resistencia en campo (ABCPPI=674-1.273). Los genotipos Libertas, 89T-123-3, Robjin, 92T-114-76, Teena, 92T-120-16 y Pollerita mostraron resistencia moderada (ABCPPI=1.3621.656). Los genotipos Sani Amarilla, Clalo, Oka y Desirée, fueron los más susceptibles (ABCPPI=2.4134.528). Los genotipos más resistentes fueron Chota Ñawi, Robusta, India (testigo), 89T-123-3 y Jaspe, que también mostraron buenos rendimientos $(0,65-0,78 \mathrm{~kg} /$ planta). Los rendimientos mas bajos $(0,08-$ $0,15 \mathrm{~kg} /$ planta) se observaron en los genotipos Libertas, 590016-7, Yema de huevo, Sani Amarilla, SH-8392-488, 88030.2, 90130.1, 88009-2 y 90145-6(9). Los genotipos DB-226(70), Libertas, Yema de huevo y Torridón mostraron bajos contenido de glucosa $\left(20-40 \mathrm{mg} \cdot \mathrm{dL}^{-1}\right)$ y alto contenido de materia seca de $22 \mathrm{a}$ 29 \%. El menor avance de verdeo se observó en Pollerita, Yema de huevo, 590016-7 y DB-226(70) con $0 \%$. Sobre la base de estos resultados se podría indicar que las variedades recomendadas para Bolivia son Robusta, Jaspe e India que ya se cultivan y los clones franceses 89T-123-3, 92T-114-76 y 92T-120-16.
\end{abstract}

Palabras clave: $P$. infestans, severidad, materia seca, ABCPPI

\section{Characterization of potato genotypes from Europe and Latin-American by resistance to late blight and culinary properties}

\begin{abstract}
The objective of the present research was to characterize 34 clones and varieties of potato of the Europe and Latin-American origin by their resistance to late blight (Phytophthora infestans) and culinary properties under field and laboratory conditions during three years. The genotypes India (check), Chota Ñawi, Cordillera, Robusta, Jaspe and Yema de huevo showed high resistance levels on field with low audpc (203-541). Genotypes 387660-10, Torridón, SH-83-92-488, 90-145-6. 94-70-2502, 89T-117-10, Pampeana, DB-226(70) and Pollerita-v showed good levels of resistance on field (audpc=674-1.273). Genotypes Libertas, 89T-123-3, Robjin, 92T-114-76, Teena, 92T-120-16 and Pollerita showed moderate resistance (audpc=1.362-1.656). Genotypes Sani Amarilla, Clalo, Oka and Desirée were the most susceptible in field (audpc $=2.413-4.528$ ). The most resistant genotypes were Chota Ñawi, Robusta, India (check), 89T-123-3 and Jaspe besides having good yield values $(0,65-0,78 \mathrm{~kg} / \mathrm{plant})$. Lower yield values
\end{abstract}

\footnotetext{
${ }^{1}$ Fundación PROINPA, C. P. 4285, Cochabamba, Bolivia. Correo electrónico:j.gabriel@proinpa.org

${ }^{2}$ CONAM, Unidad de Biodiversidad y Bioseguridad, Lima 41, Lima - Perú
} 
(0,08-0,15 kg/plant) were obtained with genotypes Libertas, 590016-7, Yema de huevo, Sani Amarilla, SH-83-92-488, 88030.2, 90130.1, 88009-2 and 90145-6(9). The genotypes DB-226(70), Libertas, Yema de huevo and Torridón showed low glucose levels (20-40 $\left.\mathrm{mg} \cdot \mathrm{dL}^{-1}\right)$, and contain from 22 to 29 percent of dry matter. The levels of advance of lower greening are expressed by genotypes Pollerita, Yema de huevo, 590016-7 and DB-226(70) with value of $0 \%$. Over basic of these resultants could be indicate that recommended varieties for Bolivia are Robusta, Jaspe and India; they are cultivating and the French clones 89T-123-3, 92T-114-76 y 92T-120-16.

Key words: $P$. infestans, severity, dry matter, AUDPC

\section{Introducción}

La papa (Solanum tuberosum L.) es uno de los cultivos alimenticios más importantes, por ser superior a todos los demás cultivos en la producción de proteína por unidad de tiempo y superficie, y en la producción de energía (Estrada, 2.000). La papa ocupa el cuarto lugar en producción mundial después del trigo, arroz y maíz. En cuanto al consumo humano ocupa el quinto lugar. Es cultivada en siete departamentos de Bolivia, considerándose un alimento fundamental en la dieta diaria de la población, se produce en diferentes nichos ecológicos, desde 1.600-4.250 msnm, es cultivada en más de 130.000 ha. El consumo per cápita es de 80 a $100 \mathrm{~kg} /$ año y es la principal fuente de alimento e ingresos de alrededor de 200.000 familias de pequeños agricultores (Estrada et al., 1994; Zeballos, 1997; Fernández-Northcote et al., 1999).

La producción de papa es afectada por diversas enfermedades. Hooker (1980) menciona 25 virus, 38 hongos, 6 bacterias, 2 micoplasmas y un viroide; además de 68 especies de nematodos, y 128 insectos-plaga, totalizando 266 patógenos y plagas. También diezman la papa factores abióticos como las heladas y la sequía. Pero de las más importantes es Phytophthora infestans que causa el tizón tardío. Esta enfermedad generalmente se controla con fungicidas, que inciden significativamente en los costos de producción, afectan al medio ambiente y la salud humana, además inducen a la aparición de razas de nuevas razas del patógeno que son resistentes a fungicidas. Por lo que es necesario considerar otras medidas de control, como el empleo de variedades resistentes, que además posean altos rendimientos y calidad culinaria aceptable para el mercado (Andrade y Revelo, 1994; Pineda y Corzo, 1994; Egúsquiza, 1994).

A pesar de la gran diversidad genética disponible en las especies silvestres de este género, sólo un pequeño número de ellas han sido utilizadas para la introgresión de caracteres de resistencia en las variedades. Se estima que apenas un $5 \%$ han sido utilizados en programas de fitomejoramiento (Gabriel et al., 1995; Colque, 1996; Estrada, 2000; Gabriel et al., 2001; Coca y Montealegre, 2006). Esto se debe, por un lado, a los problemas que existen entre determinadas especies al cruzarse. El potencial de hibridación de la papa depende, en primera instancia, de la ploidia que presente la especie silvestre o cultivada y del número de balance del endospermo (EBN). $S$. tuberosum presenta una ploidia $/ \mathrm{EBN}=4 \mathrm{x}$ (4EBN), y el potencial de hibridación es mayor con las especies 4x (4EBN) y 6x (4EBN), mientras que presenta un potencial de hibridación menor con especies 4x (2EBN) y con especies 2x (2EBN) (Spooner y Hijmans, 2001). Por otra parte en los cruzamientos, se produce la introgresión de caracteres silvestres no deseados junto con los caracteres de resistencia. Esto conlleva el desarrollo de múltiples ciclos de retrocruzamientos para eliminarlos.

Existen papas nativas cultivadas originarias de los Andes que presentan una gran variabilidad genética, entre las que se encuentran resistencias a factores abíóticos (helada, sequía) y bióticos (hongos, bacterias, virus, nematodos); además de factores de calidad. La introgresión en este germoplasma ha sido mínima y es posible que la evolución de estos cultivares continúe in situ (Gabriel y Carrasco, 1998; Ugarte et al., 1994; Estrada, 2000; Gabriel et al., 2007). 
Con el objetivo de caracterizar en Bolivia, clones y variedades de papa de origen europeo y latinoamericano, se estudió durante tres campañas agrícolas $(2.001-2.003)$ la resistencia al tizón tardío bajo las condiciones de campo y laboratorio, las características agronómicas, el comportamiento en almacén, y la calidad culinaria (materia seca y contenido de azúcares reductores) de 34 genotipos y/o variedades de papa provenientes de Holanda, Inglaterra, Francia, Argentina, Uruguay y Bolivia.

\section{Materiales y métodos}

La investigación se realizó en la comunidad de Chullchunq'ani, provincia Carrasco del departamento de Cochabamba ubicada en el km 108 sobre la carretera antigua CochabambaSanta Cruz. Geográficamente ubicada a

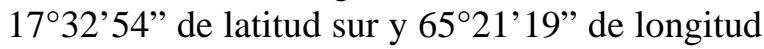
oeste, a una altitud de $2.970 \mathrm{msnm}$, con una temperatura media anual de $15{ }^{\circ} \mathrm{C}$, precipitación media anual de $629 \mathrm{~mm}$ y humedad relativa promedio del $90 \%$, la zona es montañosa con una topografía muy accidentada.

Los análisis de materia seca, azúcares reductores y verdeo se realizaron en el laboratorio de la Fundación PROINPA. Los tubérculos analizados fueron almacenados en el Centro Toralapa, ubicado en la provincia Tiraque, a $71 \mathrm{~km}$ de la ciudad de Cochabamba con una altitud de 3.430 msnm, temperatura de 8 a $15^{\circ} \mathrm{C}$ y $640 \mathrm{~mm}$ de precipitación promedio anual.

\section{Material vegetal}

Se utilizaron 34 genotipos resistentes de papa provenientes de los programas de mejoramiento genético de Holanda (CPRO-DLO), Francia (INRA), Inglaterra (SCRI), Argentina (INTA), Uruguay (INIA) y Bolivia (Fundación PROINPA). Cada país aportó con seis genotipos, la excepción fue Argentina que aportó cuatro (Tabla 1).

\section{Siembras}

En enero de 2.001, en Chullchunq'ani, Cochabamba (3.200 msnm), fueron transplantadas plántulas in vitro de 32 genotipos $\mathrm{y}$ dos testigos susceptibles, en el terreno de un agricultor para su evaluación preliminar de resistencia al tizón (Phytophthora infestans) bajo condiciones naturales de infección y para la caracterización agronómica. Las 34 accesiones fueron transplantadas en un diseño de Bloques Completos al Azar (BCA), con 14 plántulas y cuatro repeticiones. Se sembró cebada entre y alrededor de los bloques para aislar la fuente de inóculo. Cuarenta y cinco días después del transplante los primeros síntomas fueron observados y posteriormente, los genotipos fueron evaluados durante 11 semanas cada siete días con una escala porcentual. En la cosecha los genotipos fueron evaluados por rendimiento $\mathrm{y}$ tamaño de tubérculo.

En Noviembre de 2.001, en Chullchunq'ani, Cochabamba, fueron sembrados 31 genotipos $y$ cuatro testigos (dos resistentes y dos susceptibles) bajo un diseño de Bloques Completos al Azar (BCA) con cuatro repeticiones, entre cada repetición se sembró cebada como barrera viva. Se realizó una sola aplicación de fungicida al $80 \%$ de emergencia (Fitoraz) durante todo el ciclo del cultivo, las plantas fueron observadas semanalmente hasta la aparición de los primeros síntomas (77 dds). Se evaluó el avance de la enfermedad utilizando la escala internacional, donde $1=0 \%$ de daño $y$ 9=100 \% de daño.

Se realizaron nueve evaluaciones, datos que sirvieron para calcular el Area Bajo La Curva de Progreso de P. infestans (ABCPPI). A la cosecha se evaluó el rendimiento y se caracterizaron los tubérculos (tamaño, color de la piel, color de carne, profundidad de ojos, uniformidad, etc.). Se tomó cinco tubérculos de cada genotipo y se evaluó el contenido de materia seca y azúcar. En almacenamiento se evaluó el verdeo.

En diciembre de 2.003 en Chullchunq'ani fueron sembrados 31 genotipos y cuatro testigos (dos resistentes y dos susceptibles) en el terreno de un agricultor para evaluar su resistencia al tizón bajo condiciones naturales de infección y evaluación de caracteres agronómicos. Los 35 genotipos fueron sembrados en diseño de Bloques Completos al Azar (BCA) en dos surcos con 20 tubérculos por genotipo y cuatro 
repeticiones por genotipo. Alrededor de cada repetición se sembró cebada, para asegurar el aislamiento entre parcelas. Cuarenta y cinco días después de la siembra fueron observados los primeros síntomas sobre los testigos susceptibles, luego fueron evaluadas todas las accesiones durante nueve semanas en porcentaje de área de follaje afectado.

Tabla 1. Genotipos de papa provenientes de Europa y Latinoamérica

\begin{tabular}{|c|c|c|c|c|}
\hline & & Genotipos & Origen ${ }^{*}$ & $2 n$ \\
\hline \multirow[t]{6}{*}{1} & CPRO-DLO & Robjin & tbr (Rod Star x Preferent, 1926) & $4 x=48$ \\
\hline & (HOLANDA) & Libertas & tbr (Record x (Souvenir x Bato), 1946) & $4 x=48$ \\
\hline & & $94-6-147$ & $(\mathrm{axh} \times \mathrm{tbr}) \times \mathrm{tbr}$ & $2 x=24$ \\
\hline & & $94-70-2502$ & tbr x (tbr x ber) & $2 x=24$ \\
\hline & & DH 84-22-2076 & tbr & $2 x=24$ \\
\hline & & SH 83-92-488 & tbr & $2 x=24$ \\
\hline \multirow[t]{4}{*}{2} & INTA & Pampeana & MPI 59.789/12 X Huinkul MAG & $4 x=48$ \\
\hline & (ARGENTINA) & Serrana & MPI 59.703/21 x B 2.63 & $4 x=48$ \\
\hline & & OKA & S. chacoense & $2 x=24$ \\
\hline & & CLALO & S. chacoense & $2 x=24$ \\
\hline \multirow[t]{6}{*}{3} & INRA & INRA 89T.117.10 & CFQ $69.1 *$ INRA 74. 5.48 & $4 x=48$ \\
\hline & (FRANCIA) & INRA 89T.123.3 & $65 \mathrm{Z} \mathrm{A5} *$ INRA 74. 5.48 & $4 x=48$ \\
\hline & & INRA 92T.109.24 & INRA 73. $44127 *$ ASN 69.1 & $4 x=48$ \\
\hline & & INRA92T.114.76 & ARK 69.1 * Pentland Dell & $4 x=48$ \\
\hline & & INRA92T.118.5 & CEW $69.1 *$ Van Gogh & $4 x=48$ \\
\hline & & INRA92T.120.16 & CFQ $69.1 *$ INRA 73. 44.127 & $4 x=48$ \\
\hline \multirow[t]{6}{*}{4} & SCRI & Stirling & $8318(6) \times 8204 a(4)$ & $4 x=48$ \\
\hline & (INGLATERRA) & Torridón & 8373a(17) x G5833(5) & $4 x=48$ \\
\hline & & Teena & G5299(1) x Pentland Ivory & $4 x=48$ \\
\hline & & 7683a12 & $5010(13) \times 3641^{a}(81)$ & $4 x=48$ \\
\hline & & PDH247 & S. tuberosum & $2 x=24$ \\
\hline & & DB226 & S. phureja & $2 x=24$ \\
\hline \multirow[t]{6}{*}{5} & INIA & 88030.2 & Nishiyutaka x 38228416 & $4 x=48$ \\
\hline & (URUGUAY) & 88009.2 & Cupids x CIP Bulk & $4 x=48$ \\
\hline & & 90130.1 & Fredza x L. Roll Bulk & $4 x=48$ \\
\hline & & 90145.6 & Iporá x Blight Bulk & $4 x=48$ \\
\hline & & 387660.10 & Y 84.012 x Maine 47 & $4 x=48$ \\
\hline & & 590016.7 & $7 x y .1$ & $2 x=24$ \\
\hline \multirow[t]{6}{*}{6} & PROINPA & Jaspe & (sto x bre) x (tub x phu) & $4 x=48$ \\
\hline & (BOLIVIA) & Robusta & (tbr $x$ adg) $x$ tbr & $4 x=48$ \\
\hline & & Pollerita & iop x phu & $4 x=48$ \\
\hline & & Chota Ñawi & tbr x (sto x bre) & $4 x=48$ \\
\hline & & Cordillera & (tbr x adg) x (ajh x stn) & $4 x=48$ \\
\hline & & Yema de huevo & phu & $2 x=24$ \\
\hline
\end{tabular}

$*$ tbr $=$ S. tuberosum, ber $=S$. berthaultii, axh $=$ S. arnezii $\mathrm{x}$ hondelmannii, sto $=$ S. stoloniferum, bre $=S$. brevidens, adg $=$ S. andigena, phu $=$ S. phureja, iop $=$ S. iopetalum, ajh $=$ S. ajanhuiri, stn $=$ S. stenotonum .

Variables de respuesta

Área Bajo la Curva de Progreso de Phytophthora infestans (ABCPPI)

Se hicieron evaluaciones de severidad de ataque del tizón de cada genotipo a partir de la primera observación de los síntomas. Setenta y siete días después de la siembra (dds) en la campaña
2.001-2.002 y 45 días después de la siembra (dds) en la campaña 2.002-2.003. Se observaron los primeros síntomas en las variedades susceptibles, los genotipos se evaluaron durante nueve semanas a intervalos de 7 días, según la escala de evaluación descrita anteriormente. Con los datos de severidad se realizó el cálculo de 
ABCPPI (Área Bajo la Curva de Progreso de Phytophthora infestans), como medida del avance de la enfermedad a través del tiempo (Shanner y Finney, 1977).

\section{Evaluación en cosecha y poscosecha}

El segundo año de evaluación se cuantifico el número de plantas por tratamiento y para la selección de los genotipos se observaron caracteres morfológicos de uniformidad, tamaño de tubérculos, profundidad de ojos, color de piel, color de carne y sanidad de tubérculos. Se evaluó el peso (kg/planta) y número de tubérculos por categoría (primera, segunda, tercera y cuarta categoría) de cada genotipo evaluado (Tabla 2).

Tabla 2. Clasificación de tubérculos por categorías según tamaño y peso

\begin{tabular}{lcc}
\hline \multicolumn{1}{c}{ Categoría } & Tamaño (mm) & Peso (gr) \\
\hline Primera (Colque) & $>50$ & $>80$ \\
Segunda (Murmu) & $40-50$ & $60-80$ \\
Tercera (Chili) & $30-40$ & $40-60$ \\
Cuarta (Chili murmu) & $<30$ & $<40$ \\
\hline
\end{tabular}

Fuente: Fundación PROINPA, 1992.

Luego se seleccionó cinco tubérculos por tratamiento (de tamaño uniforme), que fueron colocados en sobres manila para posteriormente hacer los análisis de materia seca y verdeo en el Laboratorio de Sanidad Vegetal de la Fundación PROINPA en Cochabamba.

Una vez almacenados los tubérculos se hizo la evaluación de verdeo. Para esto se utilizó una tabla calorimétrica, tomando en cuenta el porcentaje de verdeo, intensidad de verdeo y homogeneidad en el parénquima del tubérculo. Para la determinación del avance del verdeo se usaron muestras de tubérculos cosechados, de tamaño mediano homogéneo y sin rastros de enfermedad.

\section{Análisis combinado de ABCPPI}

Para el análisis combinado del ABCPPI se consideró los datos de porcentaje de severidad de tizón de tres campañas agrícolas, y se hizo el análisis de varianza para un diseño de bloques completos al azar bajo un modelo de experimentos en serie.

\section{Evaluación de materia seca}

Para la determinación de la materia seca, se usaron muestras de tubérculos cosechados, previamente seleccionados de tamaño mediano homogéneo y sin rastros de enfermedad. Se utilizó el método de Peso Constante, que consiste en el empleo de un horno de desecación donde se introducen las muestras previamente pesadas varias veces hasta que los dos últimos valores de peso de la misma muestra pesada sean constantes a una temperatura estándar. Para este procedimiento se utilizó $4 \mathrm{~g}$ de muestra de cada tubérculo divididos en dos crisoles puestos en horno de desecación a $105{ }^{\circ} \mathrm{C}$ de temperatura inicialmente durante una hora, posteriormente se repitió el procedimiento por espacio de media hora y una media hora final, pesando las muestras consecutivamente después de haber retirado las muestras del horno comparando los dos últimos pesos que deben permanecer constantes.

\section{Determinación del contenido de azúcares}

Para la determinación del contenido de glucosa (en $\mathrm{mg} \cdot \mathrm{dL}^{-1}$ ) se realizaron mediciones con cintas de Glucostix (que sirven para medir el contenido de azúcar en la sangre), se procede a hacer un raspaje en la parte media y laterales del tubérculo para posteriormente colocar una pajilla de Glucostix en el tubérculo debajo de la muestra raspada, al cabo de 30 segundos se retiró la pajilla y se colocó en medio del papel secante presionando ligeramente durante 1 a 2 segundos. Posteriormente se esperó 90 segundos más para leer los resultados comparando con la cartilla de colores del envase. Los resultados se leyeron a simple comparación de coloración de la pajilla con la cartilla de colores expresados en $\mathrm{mg} \cdot \mathrm{dL}^{-1}$ ó $\mathrm{mmol} \cdot \mathrm{L}^{-1}$.

Los análisis de varianza y la comparación de medias fueron realizados utilizando el Proc GLM de SAS para cada una de las variables evaluadas (SAS Users Guide, 1996). 


\section{Resultados y discusión}

Análisis de varianza para ABCPPI y rendimiento

En la campaña agrícola 2.000-2.001, el análisis de varianza para ABCPPI (Tabla 3) y rendimiento (kg/planta), mostró diferencias altamente significativas entre genotipos y testigo
( $\mathrm{p}<0,01)$ para ambas variables. Los genotipos Clalo, Oka, SH-83-92-488, 92T-118-5 y Torridón fueron los más susceptibles. Los tres últimos genotipos fueron resistentes en la evaluación de foliolos, pero en campo fueron susceptibles.

Tabla 3. Análisis de varianza para ABCPPI y rendimiento (kg/planta).

Chullchunq’ani, Cochabamba, 2.000 - 2.001

\begin{tabular}{lrrlcc}
\hline \multirow{2}{*}{ Fv } & g.l. & \multicolumn{2}{c}{ ABCPPI } & \multicolumn{2}{c}{$\begin{array}{c}\text { Rendimiento } \\
\text { (kg/planta) }\end{array}$} \\
\cline { 3 - 6 } & \multicolumn{1}{c}{ C.M. } & \multicolumn{1}{c}{ F } & C.M. & F \\
\hline Genotipo & 34 & 3804699,46 & $3,22^{* *}$ & 0,0091 & $2,90^{* *}$ \\
Repetición & 3 & 84882,18 & $0,07 \mathrm{~ns}$ & 0,0015 & $0,48 \mathrm{~ns}$ \\
Error & 71 & 1182340,27 & & 0,0031 & \\
Total & 108 & & & & \\
** Significativamente diferente al Pr $<0,01$ de probabilidad
\end{tabular}

Los genotipos DB-226(70), 387660-10, Yema de huevo, Jaspe, Chota Ñawi, Robusta y Cordillera mostraron buenos niveles de resistencia en campo, así como en evaluaciones de laboratorio. En la campaña agrícola 2.001-2.002, el análisis de varianza para ABCPPI (Tabla 4) y rendimiento (kg/planta), mostró diferencias altamente significativas para ambas variables $(\mathrm{p}<$ $0,01)$ entre genotipos y testigo.

Tabla 4. Análisis de varianza para ABCPPI y rendimiento (kg/planta). Chullchunq'ani, Cochabamba, campaña agrícola 2.001-2.002

\begin{tabular}{|c|c|c|c|c|c|}
\hline \multirow{2}{*}{ Fuente } & \multirow{2}{*}{ g.l. } & \multicolumn{2}{|c|}{ ABCPPI } & \multicolumn{2}{|c|}{ Rendimiento (kg/planta) } \\
\hline & & C.M. & Valor F & C.M. & Valor F \\
\hline Genotipo & 34 & 3827910,68 & $19,97 * *$ & 0,14 & $0,5^{* *}$ \\
\hline Repetición & 3 & 531652,80 & $2,77 \mathrm{~ns}$ & 0,80 & ns \\
\hline Error & 97 & 191679,86 & & 0,02 & \\
\hline Total & 134 & & & & \\
\hline R-Cuadrado & & 0,88 & & 0,70 & \\
\hline C.V. & & 18,49 & & 47,7 & \\
\hline
\end{tabular}

**Significativamente diferente al $\operatorname{Pr}<0,01$ de probabilidad

Los genotipos Chota Ñawi, Jaspe, India (testigo), Yema de huevo, Robusta y Cordillera mostraron altos niveles de resistencia en campo con ABCPPI bajos (201-1.209), lo cual coincide con la evaluación en laboratorio.

De acuerdo con evaluaciones en laboratorio e invernadero se encontraron más genotipos resistentes en las pruebas de campo usando tubérculos semilla y no así en plántulas in vitro debido a que el efecto varietal $y$ el comportamiento del hongo no es el mismo bajo condiciones controladas que bajo condiciones de campo y su respuesta por lo tanto, está determinada por los factores ambientales que son complejos a lo largo del desarrollo y determinan el tipo de respuesta de la planta. 
Los genotipos 590016-7, 89T-123-3 y Robjin mostraron buenos niveles de resistencia en campo (ABCPPI = 1.700-1.915). Estos genotipos mostraron diferencias significativas $(\mathrm{p}<0,01)$ con relación al anterior grupo (Tabla 5).

Tabla 5. Análisis de las medias de ABCPPI de las tres campañas agrícolas evaluadas en la comunidad de Chullchunq'ani, Cochabamba, Bolivia

\begin{tabular}{|c|c|c|c|c|c|c|c|}
\hline \multirow[t]{2}{*}{ Clones } & \multicolumn{7}{|c|}{ Medias de ABCPPI* } \\
\hline & \multicolumn{2}{|c|}{ Campaña 2.000-2.001 } & \multicolumn{3}{|c|}{ Campaña 2.001-2.002 } & \multicolumn{2}{|c|}{ Campaña 2.002-2.003 } \\
\hline Desirée** & 4762 & A & 4071 & $\mathrm{AB}$ & & 1231 & ABCD \\
\hline Clalo & 4554 & $\mathrm{AB}$ & 4822 & A & & - & \\
\hline SH-83-92-488 & 4512 & $\mathrm{AB}$ & 2267 & EFGHIJK & & 340 & CDE \\
\hline Oka & 4278 & $\mathrm{AB}$ & 4294 & $\mathrm{AB}$ & & 331 & $\mathrm{DE}$ \\
\hline 92T-118-5 & 4110 & $\mathrm{ABC}$ & 2635 & CDEFGHI & & 294 & $\mathrm{DE}$ \\
\hline Torridón & 3978 & ABCD & 2653 & CDEFGHI & & 387 & $\mathrm{CDE}$ \\
\hline 7683a(12) & 3976 & ABCDE & 2638 & CDEFGHI & & 544 & BCDE \\
\hline Pollerita & 3855 & ABCDE & 3170 & BCDEFG & & 556 & BCDE \\
\hline 94-70-2502 & 3668 & ABCDE & 2125 & FGHIJKL & & 897 & BCDE \\
\hline 92T-114-76 & 3515 & ABCDEF & 2577 & CDEFGHI & & 372 & CDE \\
\hline Sani Amarilla** & 3511 & ABCDEFG & 3140 & BCDEFGH & & 1919 & A \\
\hline Stirling & 3496 & ABCDEFG & 3385 & BCDEF & & 842 & BCDE \\
\hline 90130-1 & 3484 & ABCDEFG & 2648 & CDEFGHI & & 587 & BCDE \\
\hline 92T-12016 & 3482 & ABCDEFG & 1700 & IJK & & 452 & BCDE \\
\hline 89T-123-3 & 3452 & ABCDEFG & 1897 & HIJ L & & 1292 & $\mathrm{ABC}$ \\
\hline 590016-7 & 3444 & ABCDEFG & 1748 & IJK & & - & \\
\hline Serrana & 3175 & ABCDEFGH & 3475 & BCDE & & 927 & BCDE \\
\hline PDH 247 & 3094 & ABCDEFGH & - & & & - & \\
\hline Libertas & 3076 & ABCDEFGH & 2795 & CDEFGHI & & 1090 & ABCDE \\
\hline Robjin & 2973 & ABCDEFGH & 1915 & GHIJ I & & 986 & ABCDE \\
\hline 92T-109-24 & 2862 & ABCDEFGH & 2781 & CDEFGHI & & 936 & BCDE \\
\hline Teena & 2757 & ABCDEFGH & 2644 & CDEFGHI & & 477 & BCDE \\
\hline 88030-2 & 2669 & ABCDEFGH & 3679 & $\mathrm{ABC}$ & & 407 & CDE \\
\hline 88009-2 & 2450 & ABCDEFGH & 3557 & $\mathrm{ABCD}$ & & - & \\
\hline Pampeana & 2331 & BCDEFGHI & 2324 & DEFGHIJ & & 765 & BCDE \\
\hline 89T-117-10 & 2066 & CDEFGHIJ & 2403 & DEFGHIJ & & 436 & BCDE \\
\hline Cordillera & 1832 & DEFGHIJ & 1209 & & MN & 653 & BCDE \\
\hline Robusta** & 1677 & EFGHIJ & 1041 & & MN & 451 & BCDE \\
\hline 387660-10 & 1418 & FGHIJ & 2323 & DEFGHIJ & & 1371 & $\mathrm{AB}$ \\
\hline Chota Ñawi & 1329 & FGHIJ & 201 & & $\mathrm{~N}$ & 209 & $\mathrm{E}$ \\
\hline Jaspe & 1270 & GHIJ & 680 & & $\mathrm{~N}$ & 662 & BCDE \\
\hline Yema de Huevo & 1202 & GHIJ & 1015 & & LMN & 392 & CDE \\
\hline DH-84-22-2076 & 1078 & HIJ & & & & & \\
\hline DB-226(70) & 662 & IJ & 2168 & FGHIJK & & 524 & BCDE \\
\hline India** & 938 & & & & & 278 & $\mathrm{DE}$ \\
\hline Pollerita-V** & - & & 2322 & DEFGHIJ & & 503 & BCDE \\
\hline 90145-6(9) & - & & 2680 & CDEFGHI & & 298 & DE \\
\hline
\end{tabular}

Valores seguidos por letras diferentes dentro de una columna indican diferencias significativas entre medias de acuerdo con la prueba de Tukey $(\alpha=0,05)$ *ABCPPI = Área Bajo la Curva de Progreso de Phytophthora infestans. **Testigos locales 
Desde el genotipo DB-226(70) hasta Libertas se encontraron diferentes niveles de resistencia $($ ABCPPI $=2.168-2.795)$. El primer genotipo mostró buen nivel de resistencia en campo y en laboratorio en un ensayo anterior. Estos genotipos mostraron diferencias significativas $(p<0,01)$ con el anterior grupo. No se encontraron diferencias significativas entre ellos (Tabla 5).

Los genotipos Clalo, Oka y Desirée fueron los más susceptibles en campo (ABCPPI $=4.822$, 4.294 y 4.071 respectivamente), coincidiendo estos resultados con el ensayo realizado en la campaña 2.000-2.001.

Los genotipos más resistentes Chota Ñawi, Robusta, India (testigo), 89T-123-3 y Jaspe mostraron buenos rendimientos $(0,605-0,708$ $\mathrm{kg} /$ planta). Los rendimientos más bajos fueron desde 0,15 a $0,08 \mathrm{~kg} /$ planta, en los genotipos Libertas, 590016-7, Yema De Huevo, Sani Amarilla, SH-83-92-488, 88030.2, 90130.1, 88009-2 y 90145-6(9) (Tabla 6).

En la Campaña Agrícola 2.002-2.003, hubo diferencias altamente significativas $(\operatorname{Pr}<0,01)$ entre los genotipos y los testigos para ABCPPI en Chuulchunq'ani (Tabla 5), con coeficiente de variación de 46,80\% y R-Cuadrado de 0,68. Los genotipos OKA, 90-145-6(9), 92T-118-5, India (testigo) y Chota Ñawi mostraron altos niveles de resistencia al tizón en campo con bajo ABCPPI (209-331).

Los genotipos 88030-2, Yema De Huevo, Torridón, 92T-114-76 y SH-83-92-488 mostraron buenos niveles de resistencia en campo (ABCPPI=340-407). Estos genotipos mostraron diferencias significativas $(p<0,01)$ en relación al anterior grupo.

Los genotipos Robjin, 92T-109-24, Serrana, 9470-2502, Stirling, Pampeana, Jaspe, Cordillera, 90-130-1, Pollerita, 7683 (12), DB-226(70), Pollerita(V), Teena, 92T-120-16, Robusta y 89T117-10 mostraron resistencia moderada en campo (ABCPPI=436-986).
Tabla 6. Análisis de las medias de rendimiento (kg/planta) de dos campañas agrícolas evaluadas en la comunidad de Chullchunq'ani, Cochabamba, Bolivia (*Testigos locales).

\begin{tabular}{|c|c|c|}
\hline Clon & $\begin{array}{c}\text { Campaña } \\
2.000-2.001\end{array}$ & $\begin{array}{c}\text { Campaña } \\
2.001-2.002\end{array}$ \\
\hline Desirée* & 0,025 & 0,248 \\
\hline Clalo & 0,000 & - \\
\hline SH-83-92-488 & 0,015 & 0,140 \\
\hline Oka & 0,021 & 0,000 \\
\hline 92T-118-5 & 0,012 & 0,328 \\
\hline Torridón & 0,015 & 0,218 \\
\hline $7683^{\mathrm{a}}(12)$ & 0,009 & 0,242 \\
\hline Pollerita & 0,037 & 0,226 \\
\hline $94-70-2502$ & 0,024 & 0,176 \\
\hline 92T-114-76 & 0,020 & 0,272 \\
\hline Sani Amarilla* & 0,064 & 0,145 \\
\hline Stirling & 0,024 & 0,296 \\
\hline $90130-1$ & 0,022 & 0,099 \\
\hline 92T-12016 & 0,034 & 0,368 \\
\hline 89T-123-3 & 0,022 & 0,624 \\
\hline 590016-7 & 0,009 & 0,149 \\
\hline Serrana & 0,032 & 0,404 \\
\hline PDH 247 & 0,026 & - \\
\hline Libertas & 0,013 & 0,152 \\
\hline Robjin & 0,056 & 0,337 \\
\hline $92 \mathrm{~T}-109-24$ & 0,071 & 0,242 \\
\hline Teena & 0,034 & 0,192 \\
\hline 88030-2 & 0,090 & 0,139 \\
\hline 88009-2 & 0,097 & 0,087 \\
\hline Pampeana & 0,012 & 0,251 \\
\hline 89T-117-10 & 0,049 & 0,292 \\
\hline Cordillera & 0,168 & 0,547 \\
\hline Robusta & 0,181 & 0,708 \\
\hline $387660-10$ & 0,082 & 0,201 \\
\hline Chota Ñawi & 0,182 & 0,605 \\
\hline Jaspe & 0,089 & 0,621 \\
\hline Yema de Huevo & 0,110 & 0,148 \\
\hline DH-84-22-2076 & 0,154 & - \\
\hline DB-226(70) & 0,101 & 0,427 \\
\hline $90145-6(9)$ & & 0,083 \\
\hline Pollerita-V* & & 0,211 \\
\hline India* & & 0,662 \\
\hline
\end{tabular}

Los genotipos Sani Amarilla, 387660-10, 89T123-3, Desirée, Libertas y Robjin fueron los más susceptibles en campo (ABCPPI=1919 y 1090 respectivamente). 
Estos resultados reflejan el efecto de la variedad y además el grado de adaptación de los tubérculos a condiciones de severidad en campo que se mantuvieron estables en las tres evaluaciones, confirmando así su nivel de resistencia a Phytophthora infestans.

\section{Análisis combinado del ABCPPI}

El anova combinado demostró diferencias significativas $(p<0,01)$ para genotipos $(G)$, años (A) e interacción GxA para ABCPPI (Tabla 7), con C.V. de $39,25 \%$ y $\mathrm{R}^{2}$ de 0,88 .

Tabla 7. Análisis combinado del ABCPPI para tres años

\begin{tabular}{lrrrrr}
\hline \multicolumn{1}{c}{ F.V. } & g.l. & \multicolumn{1}{c}{ S.C. } & \multicolumn{1}{c}{ C.M. } & \multicolumn{1}{c}{ F } & Pr $>$ F \\
\hline Genotipo & 37 & 156390356,51 & 4226766,39 & 12,66 & 0,0001 \\
Repetición & 3 & 1613555,02 & 537851,67 & 1,61 & 0,1876 \\
Año & 2 & 281005868,39 & 140502934,20 & 420,68 & 0,0001 \\
Genotipo x Año & 61 & 103142679,21 & 1690863,59 & 5,06 & 0,0001 \\
Error & 243 & 81158984,72 & 333897,59 & 17,83 & 0,0001 \\
Total & 346 & 694571427,97 & & & \\
R-Cuadrado & & & 0,88 & \\
C.V. & & & 39,25 & \\
\hline
\end{tabular}

Diferencias significativas al $\operatorname{Pr}<0,01$ de probabilidad

Los genotipos India (testigo), Chota Ñawi, Cordillera, Robusta, Jaspe y Yema de huevo confirmaron su alto nivel de resistencia en campo con bajo ABCPPI de 203 a 541 (Tabla 8). De acuerdo con las pruebas de laboratorio e invernadero, se encontró un mayor número de genotipos con resistencia marcada que en anteriores ensayos de campo donde se utilizaron tubérculos-semilla (pruebas en plántula).

En la Tabla 8, a pesar que los genotipos desde Pollerita-v a 387660-10 mostraron buenos niveles de resistencia en campo (ABCPPI $=674$ 1.273). Estos genotipos mostraron diferencias significativas $(\mathrm{p}<0,01)$ con relación al grupo anterior.

De Libertas a Pollerita mostraron resistencia moderada a tizón (ABCPPI=1.362-1.656). Estos genotipos mostraron diferencias significativas $(p<0,01)$ con el grupo anterior.

No se encontró ninguna diferencia significativa entre ellos. Los genotipos Sani Amarilla, Desirée, Oka, 89-123-3 y Clalo fueron los más susceptibles en campo (ABCPPI=2.413-4.528). Estos genotipos también fueron susceptibles en los tres años anteriores.
Análisis realizados con datos reportados por Francia, Inglaterra, Holanda, Bolivia, Argentina y Uruguay, sobre los mismos genotipos del presente trabajo mostraron que los genotipos más estables sin considerar el efecto ambiental fueron Robusta, Chota Ñawi, 89T-117-10, Jaspe y 95T-109-24.

\section{Tamaño y peso de tubérculos}

El análisis de varianza para tamaño y peso de tubérculo (Tabla 6), mostró diferencias altamente significativas $(\mathrm{p}<0,01)$ entre tratamientos, para tamaño y peso de tubérculos, lo que indica que los genotipos evaluados son diferentes, por lo que existen genotipos superiores para los dos factores analizados comparados con los testigos.

Se observó que los tratamientos testigo formaron tubérculos de primera categoría (Chota Ñawi, India, Pollerita y Jaspe), de igual forma que los genotipos 89T-123-3 y 92T-12016, a diferencia del resto de los genotipos evaluados.

Las medias para el número de tubérculos arrojaron diferencias significativas entre genotipos. Los testigos con valores más bajos son iguales estadísticamente a los genotipos 90145-6(9), 88009-2, Oka y Clalo (estos dos 
últimos no llegaron a formar tubérculos). El genotipo JASPE destacó con 103 tubérculos, con mayor porcentaje de tubérculos de segunda y tercera categoría. Los genotipos Yema de huevo y Chota Ñawi mostraron buen número de tubérculos (63 a 70).

El análisis de medias de la primera categoría evidenció que el genotipo Chota Ñawi fue altamente significativo (15 tub/tratamiento) respecto de los demás genotipos. Los genotipos
Clalo, Teena, Torridón y Yema de huevo fueron los que obtuvieron los valores mas bajos en este grupo. En la segunda categoría el genotipo Chota Ñawi obtuvo la media mas alta, al igual que en la categoría anterior e igual a Jaspe seguidos del genotipo 92T-12016 con valores de 30,25; 29,00 y 23,75 tub/tratamiento respectivamente. Los genotipos Robusta, India y Cordillera obtuvieron resultados sobresalientes, mientras que los genotipos Clalo, 90145-6(9), 590016-7 y OKA obtuvieron los valores más bajos.

Tabla 8. Análisis combinado de las medias para ABCPPI en tres campañas agrícolas

\begin{tabular}{|c|c|c|}
\hline Genotipos & & edias de ABCРPI* \\
\hline Clalo & 4.528 & $\mathrm{~A}$ \\
\hline Oka & 2.868 & $\mathrm{~B}$ \\
\hline Desirée** & 2.822 & $\mathrm{BC}$ \\
\hline Sani Amarilla** & 2.413 & BCD \\
\hline $88009-2$ & 2.212 & BCDE \\
\hline 88030-2 & 2.178 & BCDE \\
\hline Serrana & 2.154 & BCDEF \\
\hline 92T-109-24 & 2.032 & BCDEF \\
\hline $7683^{\mathrm{a}}(12)$ & 1.982 & BCDEF \\
\hline $90-130-1$ & 1.802 & BCDEFG \\
\hline Stirling & 1.766 & BCDEFGH \\
\hline $590016-7$ & 1.734 & CDEFGH \\
\hline $92 \mathrm{~T}-118-5$ & 1.701 & CDEFGH \\
\hline Pollerita & 1.656 & DEFGHIJ \\
\hline 89T-123-3 & 1.565 & DEFGHIJ \\
\hline Robjin & 1.518 & DEFGHIJ \\
\hline $92 \mathrm{~T}-114-76$ & 1.513 & DEFGHIJ \\
\hline Teena & 1.441 & DEFGHIJ \\
\hline $92 T-120-16$ & 1.378 & DEFGHIJ \\
\hline Libertas & 1.362 & DEFGHIJ \\
\hline $387660-10$ & 1.273 & DEFGHIJK \\
\hline Torridón & 1.227 & DEFGHIJK \\
\hline SH-83-92-488 & 1.195 & EFGHIJK \\
\hline $90-145-6$ & 1.155 & EFGHIJK \\
\hline $94-70-2502$ & 1.095 & EFGHIJK \\
\hline 89T-117-10 & 1.036 & FGHIJK \\
\hline Pampeana & 774 & GHIJK \\
\hline DB-226(70) & 718 & GHIJK \\
\hline Pollerita-V** & 674 & HIJK \\
\hline Yema de Huevo & 541 & IJK \\
\hline Jaspe & 540 & IJK \\
\hline Robusta & 532 & IJK \\
\hline Cordillera & 468 & JK \\
\hline Chota Ñawi & 446 & JK \\
\hline India** & 203 & K \\
\hline
\end{tabular}

medias de acuerdo con la prueba de Tukey $(\alpha=0,05)$. ** Testigos locales 
Para el análisis de número de medias de tubérculos en la tercera categoría se observa que el genotipo JASPE obtuvo el valor más alto con respecto al resto de los genotipos (64,75 tubérculos/tratamiento). Los genotipos Yema de huevo, DB-226(70), 387660-10, Robusta, Robjin y Cordillera mostraron ser significativamente diferentes, mientras que los genotipos 8809-2, Clalo y Oka obtuvieron los valores más bajos.

En la cuarta categoría, el genotipo Yema de huevo obtuvo mayor número de tubérculos (30,75 tub/tratamiento), en tanto que los genotipos 387660-10 y 90130.1 formaron buen número de tubérculos respecto del resto de los genotipos. Los genotipos Pollerita-v, 90145-6(9), Clalo y Oka obtuvieron los resultados más bajos para esta categoría.

Las diferencias entre genotipos para la variable de peso de tubérculos, mostró que genotipo Chota Ñawi, tuvo una media alta para peso de tubérculos (0,78 kg), seguido de Robusta, India, 89T-123-3 y Jaspe demostrando la alta resistencia de los genotipos locales al ataque del tizón, presentando los demás genotipos valores intermedios pero significativos en rendimiento.

El análisis de medias para la primera categoría mostró que el genotipo Chota Ñawi arrojó resultados sobresalientes en rendimiento para peso de tubérculos (3,49 kg/tratamiento). Los genotipos 89T-123-3, India y Pollerita mostraron diferencias significativas respecto a los demás genotipos que no llegaron a formar tubérculos y fueron significativamente iguales entre sí.

Los genotipos Chota Nawi y Jaspe destacaron en peso de tubérculos de segunda categoría obteniendo promedios significativos (3,10 y 2,51 $\mathrm{kg} /$ tratamiento) respectivamente, respecto de las medias del resto de los clones evaluados. Los genotipos ROBUSTA, 92T-12016 y 89T-123-3 obtuvieron valores sobresalientes al resto de los genotipos. Los genotipos Sani Amarilla, 94-70502, Yema de huevo, SH 83-92-488 y 90130.1 obtuvieron los valores mas bajos en rendimiento.

Para la tercera categoría los genotipos Jaspe, DB-226(70), India y Robusta obtuvieron los valores mas altos (2,05; 1,33; 1,32 y 1,28 $\mathrm{kg} /$ tratamiento) respectivamente. Los genotipos SH 83-92-488, 88009-2, Clalo, Oka mostraron los valores mas bajos para este grupo.

Como conclusión general se puede indicar que Yema de huevo, DB226 son variedades diploides $(2 n=2 x=24)$ y pertenecen a la especie Solanum phureja. Se sabe que tienen una baja resistencia horizontal al tizón y sus rendimientos son bajos. Similar situación se observó con los clones DH 84-22-2076, SH 83-92-488, PDH247 y 590016.7 que son dihaploides de $\mathrm{S}$. tuberosum $(2 n=2 x=24)$. Oka y Clalo son dos especies diploides silvestres de $S$. chacoense, que han sido reportados con resistencia a tizón, pero bajo las condiciones de Bolivia fueron susceptibles y además no produjeron tubérculos. En cambio, Jaspe, Chota Ñawi, Robusta, Cordillera e India, son mejoradas tetraploides $(2 n=4 x=48)$ con resistencia al tizón y de alto rendimiento, que han sido liberadas en Bolivia en el año 1.995 (Gabriel et al., 2001).

Los genotipos de franceses fueron los que mejor adaptación y resistencia mostraron bajo las condiciones de Bolivia y no así los otros clones evaluados que mostraron bajos rendimientos. Esto podría deberse posiblemente a que varios de los materiales evaluados fueron generados para condiciones de día largo y este aspecto influyo significativamente en el rendimiento. Los agricultores en Bolivia desean variedades de color rojo o rosado, por lo cual varios de los clones franceses cumplen este requisito y no así varios de los clones evaluados que en general son de color crema, por lo que estos no podrían ser cultivados; sin embargo podrían ser utilizados como progenitores en cruzamientos.

Los genotipos Sani Amarilla (Solanum andigena), 387660-10, 89T-123-3, Desirée, Libertas y Robjin fueron los más susceptibles al tizón tardío.

\section{Análisis de verdeo}

Los niveles de avance de verdeo más bajos (0 \%) se encontraron en los genotipos Pollerita, Yema de huevo, 590016-7 y DB-226(70) a los treinta 
días después de la cosecha. Estos genotipos pueden ser almacenados por más tiempo. El verdeo está íntimamente relacionado a la producción de clorofila y solanina, que son sustancias químicas que se producen en el tubérculo a consecuencia del almacenamiento bajo condiciones de temperatura $\left(8-10{ }^{\circ} \mathrm{C}\right)$, luz (difusa a oscura) y humedad apropiadas (90\% de humedad), como a la producción de glicoalcaloides que son sustancias tóxicas que impiden su consumo y evitan el ataque de plagas. Cuando las condiciones mencionadas se alteran, sobre todo la humedad, pueden ser susceptibles al ataque de polillas, hongos como el t’octu o k’anura, pudrición seca y pudrición blanda (Gull y Isenberg, 1960).

Los genotipos 92T-109-24, Teena, Robjin, 9470-2502, Robusta, Torridón, 387660-10, 880092, India, Stirling, 7683a(12), Pampeana, Desirée, 88030.2, 90130.1 y 89T-123-3 mostraron niveles intermedios del avance de verdeo (50\%), manteniendo sus características para consumo por un periodo razonable y manteniendo su disponibilidad y viabilidad para próximos periodos agrícolas. Los genotipos 92T-118-5, 90145-6(9) y Jaspe, mostraron un avance de verdeo del $100 \%$.

Tabla 9. Contenido de glucosa $\left(\mathrm{mg} \cdot \mathrm{dL}^{-1}\right)$ y porcentaje de materia seca en tubérculos después de dos semanas de la cosecha

\begin{tabular}{lccc}
\hline \multicolumn{1}{c}{ Genotipo } & $\begin{array}{c}\text { Glucosa } \\
\left(\mathrm{mg} \cdot \mathrm{dL}^{-1}\right)\end{array}$ & \% de materia seca & P. E. (kg) \\
\hline 92T-109-24 & 800 & 28,75 & 1,14 \\
DB-226(70) & 20 & 28,75 & 1,14 \\
Robjin & 400 & 26,00 & 1,11 \\
Libertas & $20-40$ & 25,50 & 1,10 \\
SH-83-92-488 & $>800$ & 23,25 & 1,09 \\
Chota Ñawi & 800 & 22,75 & 1,09 \\
Jaspe & 140 & 22,50 & 1,10 \\
Cordillera & $70-100$ & 22,25 & 1,09 \\
Torridón & $20-40$ & 22,00 & 1,09 \\
94-70-2502 & 800 & 21,50 & 1,09 \\
Yema de Huevo & 20 & 21,50 & 1,09 \\
90130-1 & $40-70$ & 21,25 & 1,09 \\
92T-12016 & 110 & 21,25 & 1,09 \\
Teena & 250 & 21,00 & 1,09 \\
Pampeana & 250 & 21,00 & 1,09 \\
Robusta & $40-70$ & 21,00 & 1,09 \\
92T-114-76 & $400-800$ & 20,75 & 1,08 \\
387660-10 & 140 & 20,75 & 1,08 \\
88009-2 & $250-400$ & 20,50 & 1,08 \\
Desirée & 140 & 20,25 & 1,08 \\
88030-2 & 800 & 20,00 & 1,08 \\
90145-6(9) & 110 & 19,75 & 1,08 \\
89T-117-10 & 250 & 19,25 & 1,08 \\
590016-7 & $400-800$ & 19,25 & 1,07 \\
7683a(12) & $70-110$ & 18,75 & 1,07 \\
Pollerita & 40 & 18,50 & 1,07 \\
Pollerita-V & 110 & 18,50 & 1,07 \\
India & 180 & 18,50 & 1,07 \\
92T-118-5 & 800 & 17,50 & 1,07 \\
Serrana & $250-400$ & 17,25 & 1,07 \\
Stirling & 180 & 16,75 & 1,06 \\
89T-123-3 & 800 & 16,00 & 1,06 \\
Sani Amarilla & $400-800$ & 14,00 & 1,06 \\
\hline
\end{tabular}

P.E. = Peso específico, $\quad\left(\mathrm{mg} \cdot \mathrm{dL}^{-1}\right)=$ miligramos por decilitro 
Análisis de glucosa y materia seca

Los genotipos DB-226(70), Libertas, yema de huevo y Torridón mostraron los niveles más bajos de glucosa $\left(20-40 \mathrm{mg} \cdot \mathrm{dL}^{-1}\right)$ lo que demuestra su potencial para ser procesados en la agroindustria como la fabricación de hojuelas o papas fritas (Tabla 9).

Los niveles altos de glucosa fueron encontrados en los genotipos 92T-109-24, 92T-118-5, Serrana, 92T-114-76, SH83-92-488(9), Chota Ñawi, 88030.2, 590016-7 y Sani Amarilla (400$\left.800 \mathrm{mg} \cdot \mathrm{dL}^{-1}\right)$. Los contenidos intermedios de glucosa (40-70 $\mathrm{mg} \cdot \mathrm{dL}^{-1}$ ) se encontraron en los genotipos Pollerita, Robusta y 90130.1.

El análisis de materia seca mostró que los genotipos DB-226(70), Libertas, Yema de huevo y Torridón contienen 22 a $29 \%$ que coincide con los niveles más bajos de glucosa. Los genotipos 92T-109-24, 92T-118-5, Serrana, 92T-114-76, SH83-92-488(9), Chota Ñawi, 88030.2, 5900167 y Sani Amarilla poseen un porcentaje de materia seca entre 18 a 21. También se pudo evidenciar que el peso específico mostró resultados igualmente altos, con valores de (1.086-1.142 kg/tub) en los mismos genotipos DB-226(70), Libertas, Yema de huevo y Torridón. Los valores intermedios de peso específico homólogos a los genotipos desde 92T109-24 hasta Sani Amarilla arrojaron valores de $1.065-1.086 \mathrm{~kg} / \mathrm{tub}$.

Estudios recientes en Bolivia con los cultivares Robusta, India, Cordillera y Jaspe mostraron buenas características culinarias para su transformación en agroindustria.

DB-226(70) y Yema de huevo pertenecen a la especie diploide $S$. phureja, por lo que sus rendimientos son muy bajos, tal como se observó en los tres años de evaluación. En cambio Libertas de Holanda y Torridón de Inglaterra, si bien son tetraploides, tuvieron bajos rendimientos y baja resistencia al tizón, esto posiblemente debido a que estas variedades fueron generadas para días largos y no para las condiciones de día corto, que es el caso de Bolivia.

\section{Agradecimientos}

A la Unión Europea que financió el proyecto ECOPAPA [Enrichment of Potato Breeding Programs in Latin America and Europe with Resistance to Late Blight, Phytophthora infestans (ERBIC18 CT98 0318)], ejecutado en Bolivia y otros países, cuyos resultados se reportan en el presente documento.

\section{Literatura Citada}

Andrade H. y Revelo, J. 1994. Breve diagnóstico de la Problemática del cultivo de la papa con énfasis en Resistencia a Enfermedades. En: Broers, L.H.M. (ed.). Resistencia Duradera en Cultivos Alto Andinos. Memorias 1er taller sobre Resistencia Duradera. Mayo 30-Junio 10 de 1.994. Quito, Ecuador. p. 79-83.

Coca, M. y Montealegre, N. 2006. Short communication. Resistance to Phytophthora infestans in populations of wild potato species in the Sorata microcentre of genetic diversity, La Paz, Bolivia. Spanish Journal of Agricultural Research 4(2): 156-160

Colque, C. 1996. Caracterización de genes de virulencia en poblaciones de Phytophthora infestans (Mont.) de Bary, que infectan a la papa y determinación de la resistencia en Solanum spp. Univ. Mayor de San Simón. Cochabamba, Bolivia. 223 p.

Egúsquiza, R. 1994. Resistencia Genética Duradera en el Cultivo de la papa en el Perú. En: Broers, L.H.M. (ed.). Resistencia Duradera en Cultivos Alto Andinos, Memorias 1er taller sobre Resistencia Duradera. Mayo 30-Junio 10 de 1.994. Quito, Ecuador. p. 89-93.

Estrada, R.N. 2000. La biodiversidad en el mejoramiento genético de la Papa. Centro Internacional para el Desarrollo (CID), PROINPA, CIP. La Paz, Bolivia. 372 p.

Estrada, R.N. 1974. Mejoramiento genético de la papa resistencia a enfermedades. En: Nuevos enfoques para el mejoramiento genético en papa 
para la Subregión Andina, Quito, Ecuador. p. 4655

Fernández-Northcote, E.; Navia, O. y Gandarillas, A. 1999. Bases de las Estrategias de Control Químico del Tizón Tardío de la Papa Desarrolladas por PROINPA en Bolivia. Revista ALAP 1 (11): 1-25.

Fernández-Northcote, E. y Plata, G. 1998. Bases para una resistencia duradera al tizón mediante la utilización de cultivares de papa con genes- $\mathrm{R}$ vencidos dentro de un manejo integrado. En: Compendio de exposiciones de la XVIII Reunión de la Asociación Latinoamericana de la Papa. Cochabamba, Bolivia. p. 83-84.

Gabriel, J. y Carrasco, E. 1998. Evaluación preliminar de la resistencia durable al tizón Phytophthora infestans en cultivos nativos de papa del banco de germoplasma boliviano. En: Danial, D. (ed.). Segundo Taller de Resistencia Duradera en Cultivos Altos en la zona Andina "PREDUZA" 22 al 24 de sep. Cochabamba Bolivia. p. 153-158

Gabriel, J.; Carrasco, E.; García, W., Equize, H.; Thiele, G.; Torrez, R.; Ortuño, N.; Navia, O.; Franco, J. y Estrada, N. 2001. Experiencias y Logros sobre Mejoramiento convencional y selección participativa de cultivares de papa en Bolivia. Revista ALAP 1: 169-192

Gabriel, J., Castillo, F., Sosa, C. y Rivera-Peña, A. 1995. Resistencia de híbridos interespecíficos de papa resistentes al tizón tardío (Phytophthora infestans (Mont.) De Bary). Revista de Agricultura 26: 38-40

Gabriel, J.; Coca, A.; Plata, G. y Parlevliet, J.E. 2007. Characterization of the resistance to Phytophthora infestans in local potato cultivars in Bolivia. Euphytica 153:321-328

Gonzáles-Herrero, F. 2000. Producción de Patatas. En: Pascualena, J. y Ritter, E. (ed.)
Libro de Actas del Congreso Iberoamericano de Investigación y Desarrollo en Patata. Patata 2.000, Julio 3-6, 2.000, Vitoria-Gasteiz. España. p. 378-395

Gull, D. e Isenberg, M. 1960. Chlorophyll and solanine content and distribution in four varieties of potatoes tuber. Am. Soc. Hort. Sci. 75:546-556.

Hooker, W.J. 1980. Compendio de enfermedades de la papa. Nematodos parásitos de la papa. CIP. Lima. Perú. p. 131-134.

Pineda, R. y Corzo, P. 1994. Desarrollo y Mejoramiento Genético del cultivo de la papa en Colombia. En: Broers, L.H.M. (ed.). Resistencia Duradera en Cultivos Alto Andinos, Memorias 1er taller sobre Resistencia Duradera. Mayo 30Junio 10 de 1.994. Quito, Ecuador. p. 83-89.

SAS Institute Inc. 1996. SAS/STAT Users Guide, Version 6, Fourth Edition, Vol. 2, SAS Institute Inc., Cary, N.C.

Shanner, G. y Finney, R. E. 1977. The effect of nitrogen fertilization on the expression of slowmildewing resistance in knox wheat. Phytopathology 67: 1051-1056.

Spooner, D.M. y Hijmans, J. 2001. Potato systematics and germplasm collecting, 19892.000. American Journal of Potato Research 78: 237-268.

Ugarte, M. L.; Estrada, N.; García, W. y Carrasco, E. 1994. Identificación, evaluación, caracterización y conservación del germoplasma de papa, raíces y tubérculos andinos en Bolivia. En: Céspedes, L., Perpich, C. (ed.). Primera Reunión Internacional de Recursos genéticos de papa, raíces y tubérculos andinos. IBTA. p. 295

Zeballos, H. 1997. Aspectos Económicos de la Producción de papa en Bolivia. COSUDE, CIP (Centro Internacional de la Papa). Lima, Perú. 